\title{
锌配体化合物选择性诱导、稳定、菼光检测 G-四联体
}

\author{
王雅芬 ${ }^{\dagger}$ 张雄 $^{\dagger}$ 刘朝兴 $^{\dagger}$ 周翔* \\ (武汉大学 化学与分子科学学院 生物医药高分子教育部重点实验室 高等研究院 湖北省过敏及免疫相关疾病
}

重点实验室 武汉 430072)

\begin{abstract}
摘要 通过两步简单的合成反应, 制备了锌的配体化合物(ZSC). 并将其与不同构型的 G-四联体作用, 发现其可以很好 的选择性苂光打开检测 G-四联体. 通过荧光, 圆二色谱 $(\mathrm{CD})$ 等实验的深入研究, 表明这个化合物兼具诱导、稳定和苂 光打开检测 $\mathrm{G}$-四联体的优点. 这是第一次报道既可以诱导稳定 $\mathrm{G}$-四联体，又可以进行荧光打开检测定量分析 $\mathrm{G}$-四联 体的锌的 salen 配体复合物. 此外, 这个化学试剂还可以将 $\mathrm{Z}$ 型 G-四联体转变成平行的 G-四联体. 这是一个针对 G-四 联体的选择性的、敏感的和拓扑特异性的探针. 这个探针与不同 G-四联体的序列之间的相互作用的研究, 展示了它可 能作为一种抗癌药物的潜力, 并且, 有望将其用于基于 G-四联体的检测方法检测其他生物物质的领域.

关键词＼cjkstart锌配体; 选择性; G-四联体; 菼光; 稳定性
\end{abstract}

\section{Induction Stabilization and Fluorescence-based Switch-on Detection of G-Quadruplex by Zinc(II)-salen Complex}

\author{
Wang, Yafen $^{\dagger} \quad$ Zhang, Xiong $^{\dagger} \quad$ Liu, Chaoxing $^{\dagger} \quad$ Zhou, Xiang* $^{*}$ \\ (College of Chemistry and Molecular Sciences, Key Laboratory of Biomedical Polymers of Ministry of Education, The Insti- \\ tute for Advanced Studies, Hubei Province Key Laboratory of Allergy and Immunology, Wuhan University, Wuhan 430072)
}

\begin{abstract}
G-quadruplexes play vital roles in telomere maintenance and other biological systems. An effective G-quadruplexes stabilizer can be a promising medicine for cancer therapy. Here, a Zinc(II) with salen derivatives as ligands (ZSC) has been prepared through two simple steps syntheses. 2,4-Dihydroxybenzaldehyde (1 equiv.), 1-(2-chloroethyl)pyrrolidine hydrochloride (1 equiv.) and potassium carbonate (2 equiv.) were dissolved in $150 \mathrm{~mL}$ acetone. After reflux for $20 \mathrm{~h}$ in Ar atmosphere and purification, the product 2-hydroxy-4-(2-(pyrrolidin-1-yl)ethoxy)benzaldehyde was obtained to yield $3.24 \mathrm{~g}(68.8 \%)$ as a pale solid. For the other step, 2-hydroxy-4-(2-(pyrrolidin-1-yl)ethoxy)benzaldehyde ( 2 equiv.) and diaminomaleonitrile ( 1 equiv.) were dissolved into $10 \mathrm{~mL}$ methanol. After 20 min stirring at $60{ }^{\circ} \mathrm{C}$ in dark atmosphere, the Zinc acetate dehydrate (1 equiv.) was added in the solution for further $2 \mathrm{~h}$ reaction time. The final product Zinc(II)-salen Complex (ZSC) was purified to yield $245 \mathrm{mg}(67.7 \%)$ as a red powder. The fluorescence, CD spectra are reported, giving insight into the intrinsic properties of the compound. The Zinc(II)-salen Complex could simply discriminate G-quadruplex from other DNA conformations such as hairpin, double-stranded DNA and single-stranded DNA by using fluorescence spectra. To the best of our knowledge, ZSC is the first Zinc(II)-salen Complex bearing features of inducing, stabilizing, fluorescence-based switch-on detecting G-quadruplex. In addition, ZSC can also change the Z G-quadruplex to parallel G-quadruplex. Such a sensitive and topology-specific probe is able to light up G-quadruplexes and gain an excellently quantitative detection of DNA bearing G-quadruplexes sequences. The detailed study of the interactions of ZSC with different topology DNAs has allowed us to build the most vital features that Zinc(II)-salen Complex can be a potential anticancer drug and in application to other bioanalytes. In the future, we will use the Zinc(II)-salen Complex to downstream the gene expression in the gene promoter area to help analyze more biological processes or use it to selectively inhibit viruses which containing G-quadruplexes.
\end{abstract}

Keywords Zinc(II)-salen complex; selectivity; G-quadruplex; fluorescence; stability

\section{1 引言}

高选择性的荧光打开检测 (Fluorescence-based Switch-on Detection)核酸是表观遗传学中很重要的研究 领域之一．荧光信号从无到有，可以非常有效地降低检
测背景，从而实现对目标物的高灵敏性的定性和定量分 析 ${ }^{[1]}$. 对于单个修饰碱基而言, 比如醛基修饰嘧啶的检 测，可以通过设计合成有机小分子化合物与目标检测物 发生共价反应从而实现苂光的打开检测 ${ }^{[2,3]}$. 然而, 针对

\footnotetext{
* E-mail: xzhou@whu.edu.cn

${ }^{\dagger}$ These authors contributed equally to this work.

Received April 13, 2017; published April 28, 2017.

Supporting information for this article is available free of charge via the Internet at http://sioc-journal.cn.

Project supported by the National Natural Science Foundation of China (Nos. 21432008, 91413109, 21672165 and 21672167).

项目受国家自然科学基金(Nos. 21432008,91413109, 21672165 and 21672167)资助.
} 
不同的核酸二级结构, 比如 G-四联体, 则需要通过控制 微观环境的亲疏水性、氢键、静电作用等实现对目标核 酸的识别与调控 ${ }^{[4]}$. G-四联体在人体端粒, 核糖核苷酸, 肝癌病毒, 埃博拉病毒中均有发现 ${ }^{[5 \sim 8]}$. 检测识别调控 G-四联体对人类延长寿命, 疾病诊断治疗具有重大的意 $义^{[9]}$. 在端粒的富 $G$ 序列中容易形成 G-四联体. 通过设 计合成具有特异性靶向原癌基因启动子区域的 G-四联 体序列的 G-四联体稳定剂, 可以非常有效地调控癌基

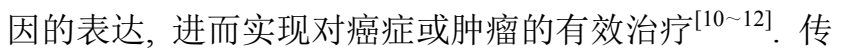
统 G-四联体的稳定剂可以分为三类. 一是显著地增加 G-四联体的稳定性, 本身不具备荧光检测作用. 比如喹 啉衍生物, 卟啉衍生物, 吡啶酰胺衍生物, 钉配体衍生 物, 镍配体衍生物等 ${ }^{[13 ~ 19]}$. 二是荧光标记 G-四联体. 化 合物本身既具有荧光, 又能够实现对 G-四联体的荧光

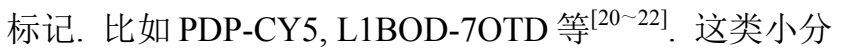
子的合成设计分为稳定 $\mathrm{G}-$ 四联体的部分和苂光标记部 分. 三是荧光打开检测 G-四联体, 硫黄素 $\mathrm{T}$, 咪唑衍生

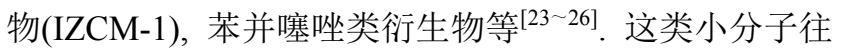
往设计比较巧妙, 既可以选择性作用于 G-四联体, 又可 以发生荧光响应, 具有很广泛的应用前景. 比如用来检 测蛋白酪氨酸激酶 7 (PTK7) ${ }^{[27]}$ 和通过构建 G-四联体与 探针体系进行更多识别和检测等的运用 ${ }^{[28 ~ 31]}$.

本文主要在我们课题组前期工作的基础上, 通过精 心的设计篮选, 首次报道一个具有诱导稳定并且苂光打 开 G-四联体的锌的配体化合物 Zinc(II)-salen complex (ZSC). 并且通过运用荧光检测器和圆二色谱等仪器详 细研究其与不同构型的 G-四联体之间的相互作用.

\section{2 结果与讨论}

\section{1 化合物的合成}

我们先将 2, 4-二羟基苯甲醛与 $N$-(2-氯乙基)吡咯烷 盐酸盐在碱性条件下反应生成 2-差基-4-(2-吡咯烷基)乙 氧基苯甲醛; 然后通过 2,3-二氨基-2-丁烯二腈与其形成 席夫碱, 再加入醋酸锌配位形成最终产物(图 1). 该反应 设计简单，步骤少，反应产率高.

\section{2 不同序列荧光响应}

为了验证化合物 ZSC 的苂光性质, 我们将化合物 和已经报道在 $\mathrm{K}^{+}$存在的情况下会形成 $\mathrm{G}$-四联体的脱氧 核糖核苷酸序列和不会形成 $\mathrm{G}$-四联体的核酸序列(单 链、双链以及具有发卡结构的 DNA $)^{[26,32]}$ 进行孵育, 通 过苂光强度的变化进行探索. 首先我们尝试了反应缓冲 液中有 $100 \mathrm{mmol} / \mathrm{L} \mathrm{K}^{+}$存在的情况下苂光强度的变化, 将相同浓度的不同 DNA 序列和 $5 \mu \mathrm{mol} / \mathrm{L}$ 的 ZSC 在 $37{ }^{\circ} \mathrm{C}$ 条件下捊育 $30 \mathrm{~min}$ 后进行苂光光谱的测试. 如图 2a 所示, 当化合物遇到具有 G-四联体结构的 DNA 后苂 光显著增强, 而在体系中加入单链、双链或者发卡结构 的 DNA 时荧光变化小. 值得注意的是, 混合型的 G-四
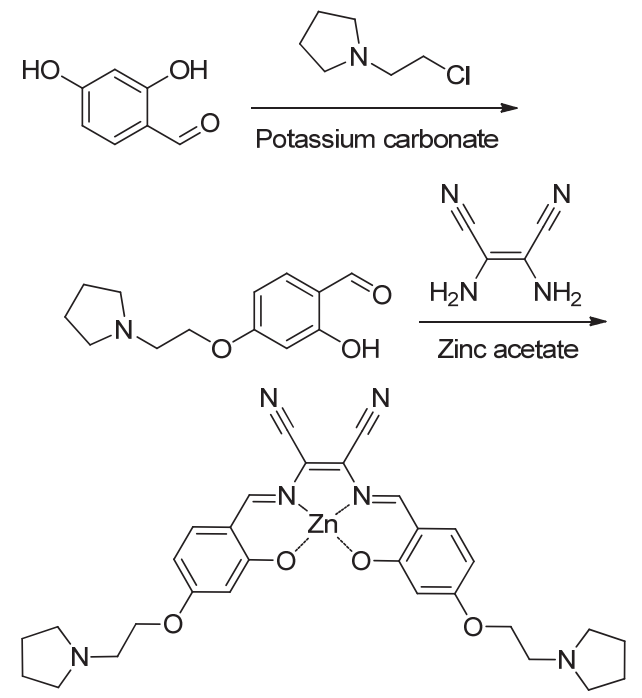

图 1 小分子化合物的合成路线

Figure 1 Synthesis of Zinc(II)-salen complex (ZSC)

Step 1: 2,4-dihydroxybenzaldehyde (2.76 g, $20 \mathrm{mmol})$, 1-(2-chloroethyl)pyrrolidine hydrochloride $(3.40 \mathrm{~g}, 20 \mathrm{mmol})$ and potassium carbonate $(5.56 \mathrm{~g}$, $40 \mathrm{mmol}$ ) were dissolved in $150 \mathrm{~mL}$ acetone. After reflux for $20 \mathrm{~h}$ in Ar atmosphere, the product was dried in vacuum, then extraction by ethyl acetate $(200 \mathrm{~mL})$ three times. Finally, the product 2-hydroxy-4-(2-(pyrrolidin1-yl)ethoxy)benzaldehyde was purified by silica gel column chromatography using eluent $\mathrm{CH}_{2} \mathrm{Cl}_{2} / \mathrm{MeOH}(V / V=30: 1 \sim 20: 1)$ to yield $3.24 \mathrm{~g}(68.8 \%)$ as a pale solid. Step 2: 2-hydroxy-4-(2-(pyrrolidin-1-yl)ethoxy)benzaldehyde $(285 \mathrm{mg}, 1.2 \mathrm{mmol})$ and diaminomaleonitrile $(65 \mathrm{mg}, 0.6 \mathrm{mmol})$ were dissolved into $10 \mathrm{~mL}$ methanol. After $20 \mathrm{~min}$ stirring at $60{ }^{\circ} \mathrm{C}$ in dark atmosphere, the Zinc acetate dehydrate $(132 \mathrm{mg}, 0.6 \mathrm{mmol})$ was added in the solution for further $2 \mathrm{~h}$ reaction time. The final product Zinc(II)-salen Complex (ZSC) was obtained by silica gel column chromatography using eluent $\mathrm{CH}_{2} \mathrm{Cl}_{2} / \mathrm{MeOH} / \mathrm{Et}_{3} \mathrm{~N}(V / V / V=65: 3: 1)$ to yield $245 \mathrm{mg}(67.7 \%)$ as a red powder.

联体苂光强度变化最为显著. 得到这一结果后, 我们将 反应体系换成无 $\mathrm{K}^{+}$的状态，从图 $2 \mathrm{~b}$ 可以看到，在没有 $\mathrm{K}^{+}$的时候, 化合物和 $\mathrm{G}$-四联体孵育后荧光增强的倍数 比有 $\mathrm{K}^{+}$时更显著, 而其他的 DNA 序列荧光增强依然不 明显. 我们猜测是因为 $\mathrm{ZSC}$ 和 $\mathrm{K}^{+}$与 $\mathrm{G}$-四联体作用有一 个竞争过程, 当有 $\mathrm{K}^{+}$存在时, ZSC 只有部分可以作用于 $\mathrm{G}-$ 四联体的平面，在相同浓度的 DNA 下，没有 $\mathrm{K}^{+}$时 ZSC 可以自由的作用于 G-四联体的平面, 从而荧光增 强的更多. 从这个结果初步判断 ZSC 具有诱导 G-四联 体形成的功能. 进一步的验证我们将通过圆二色谱进行 证实.

\subsection{ZSC 与混合型 G-四联体 DNA 的相互作用的研究}

图 2 的结果表明 UspBQ1 与化合物 ZSC 作用后荧 光强度显著增强, 而荧光的强度是否与 DNA 的浓度呈 正相关对于定量是很关键的，因此不同浓度的 UspBQ1 与化合物 ZSC 进行了孵育, 图 $3 \mathrm{a}$ 和图 $3 \mathrm{~b}$ 结果表明随着 DNA 浓度的增强荧光强度是依次增高的. 这对 G-四联 体的定量提供了一定的基础. 为了进一步验证化合物 ZSC 是否可以诱导 G-四联体的形成, 我们利用圆二色 谱做了进一步的研究. 圆二色谱是表征核酸二级结构的 有效方式. 通过 $\mathrm{CD}$ 光谱可以有效地判断 G-四联体是属 

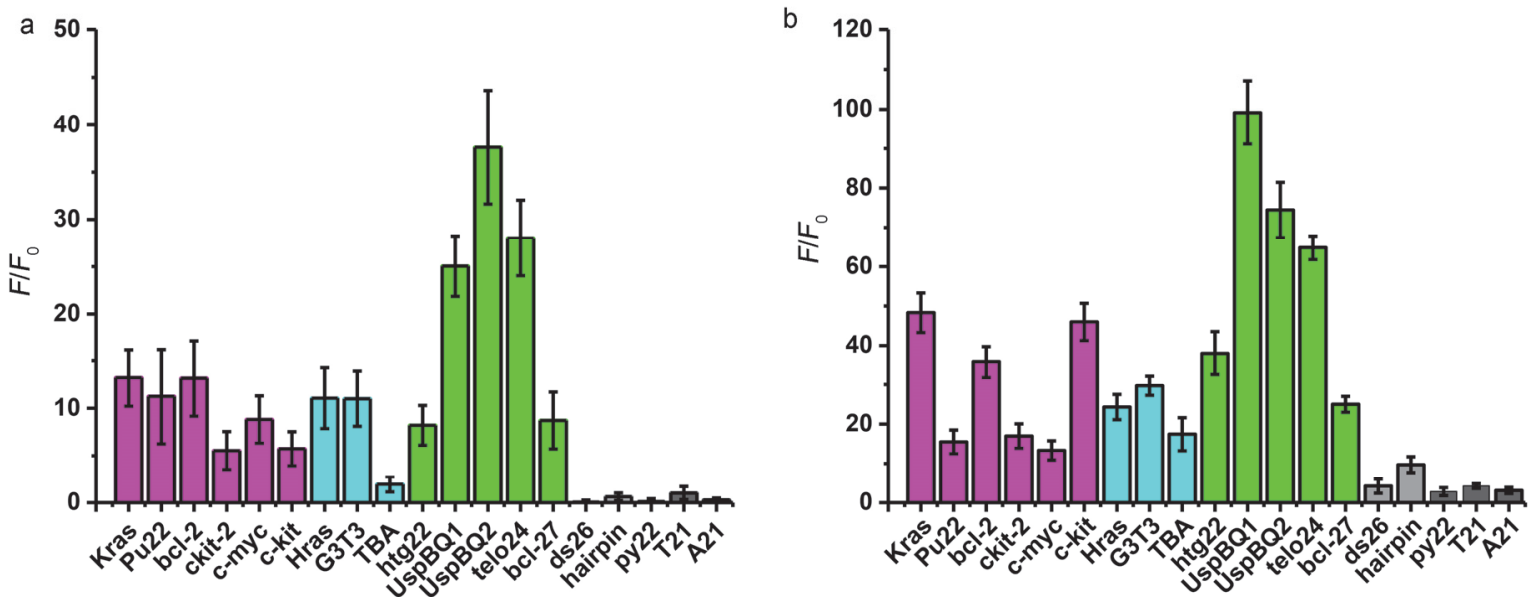

图 2 不同构型的 DNA 序列与化合物 ZSC 的苂光响应

Figure 2 Fluorescence spectra of different configuration ODNs after incubation with ZSC

a. The mixture of $1 \mu \mathrm{L}$ DNA $(100 \mu \mathrm{mol} / \mathrm{L})$ and $10 \mu \mathrm{L}$ ZSC $(200 \mu \mathrm{mol} / \mathrm{L})$ were incubated in $100 \mathrm{mmol} / \mathrm{L} \mathrm{K}$ Tris- $\mathrm{HCl}(10 \mathrm{mmol} / \mathrm{L}$, $\mathrm{pH} 7.2)$ buffer at $37{ }^{\circ} \mathrm{C}$ for 30 min. b. The mixture of $1 \mu \mathrm{L} \mathrm{DNA}(100 \mu \mathrm{mol} / \mathrm{L})$ and $10 \mu \mathrm{L} \mathrm{ZSC}(200 \mu \mathrm{mol} / \mathrm{L})$ were incubated in $10 \mathrm{mmol} / \mathrm{L} \mathrm{Tris}-\mathrm{HCl}(\mathrm{pH} 7.2)$ buffer at $37{ }^{\circ} \mathrm{C}$ for $30 \mathrm{~min}$
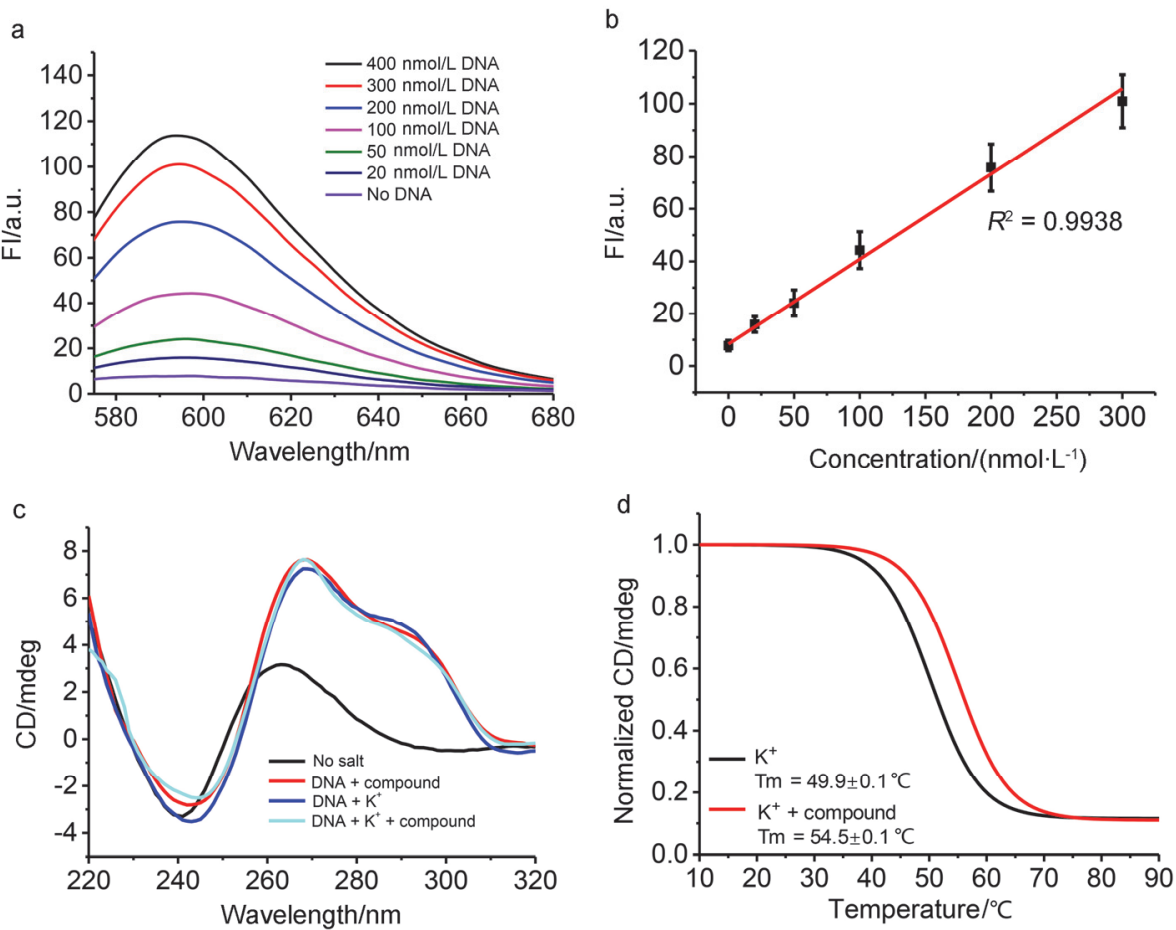

图 3 混合型 $\mathrm{G}$-四联体(UspBQ1)的荧光线性图和 $\mathrm{CD}$ 分析

Figure 3 Fluorescence intensity and CD analysis of hybrid-type G-quadruplex UspBQ1

a. The fluorescence spectra of UspBQ1 in different concentration incubated with $10 \mu \mathrm{mol} / \mathrm{L} \mathrm{ZSC}$ under the condition of $10 \mathrm{mmol} / \mathrm{L}$ Tris- $\mathrm{HCl}(\mathrm{pH} 7.2)$ at $37{ }^{\circ} \mathrm{C}$ for 30 min. b. Analysis of data about fluorescence intensity dependence on different DNA concentration shown in (a). c. CD spectra of UspBQ1 under different condition. d. The melting curves of UspBQ1 under the condition of $100 \mathrm{mmol} / \mathrm{L} \mathrm{K}^{+}$and $100 \mathrm{mmol} / \mathrm{L} \mathrm{K}^{+}$with $10 \mu \mathrm{mol} / \mathrm{L} \mathrm{ZSC}$ respectively

于平行结构、反平行结构、混合型结构或者是 Z-型结 构 ${ }^{[33]}$. 在 $100 \mathrm{mmol} / \mathrm{L} \mathrm{K}^{+}$存在的情况下, UspBQ1 在 266 $\mathrm{nm}$ 处和 $290 \mathrm{~nm}$ 处分别出现了一个正的尖峰和宽峰, 这 些是混合型 $\mathrm{G}$-四联体的特征峰, 在 ZSC 的存在下 UspBQ1 同样地出现了混合型 G-四联体的特征峰. 当 $\mathrm{K}^{+}$和 $\mathrm{ZSC}$ 同时存在时光谱图也没有发生变化(图 3c). $\mathrm{ZSC}$ 是否可以稳定 $\mathrm{G}$-四联体也是至关重要的, 从图 3d
可以看到，当化合物 ZSC 加入到体系后，UspBQ1 的熔 解温度(TM)上升了 $4.6{ }^{\circ} \mathrm{C}$. 这一结果表明化合物 ZSC 不仅能够诱导 $\mathrm{G}$-四联体的形成而且可以稳定 G-四联体. 同时，我们还将化合物与另一条混合型 $\mathrm{G}$-四联体进行 作用. 同样地我们发现, 化合物 ZSC 可以有效地诱导 UspBQ2 形成混合型 G-四联体. 并且 DNA 的浓度与荧 光有一个很好的线性关系(补充数据图 S1). 为了研究小 
分子与 G-四联体的结合情况, 我们通过 Job'S Plot 实验 探讨化合物和 G-四联体的最佳结合配比. 配制一系列 不同比例浓度的 ZSC 与 DNA 体系进行苂光光谱的测定. 通过分析可以看到当 ZSC 与 DNA 的物质的量浓度比为 $1: 1$ 时, 荧光强度最强. 从实验数据上看, 化合物 ZCS 与 $\mathrm{G}$-四联体是 $1: 1$ (物质的量浓度比)结合的(支持信息 图 S3).

\section{$2.4 Z S C$ 与平行 G-四联体 DNA 的相互作用的研究}

原癌基因启动子 ckit 含有可以形成平行 G-四联体 的 DNA 序列, 它的生物学功能可能与癌症息息相关 ${ }^{[34]}$. 我们将一种平行的 G-四联体 ckit 序列与化合物 ZSC 进 行了研究. 首先对荧光强度与 ckit 的浓度的关系做了一 系列实验, 从图 $4 \mathrm{a}$ 和图 $4 \mathrm{~b}$ 可以看到体系的苂光强度是 随着 ckit 的浓度增加而增强. 其次为了了解化合物 ZSC 对 ckit 的结构是否有影响, 在圆二色谱的测定实验 中, $\mathrm{K}^{+}$和 ZSC 分别加入 ckit 里, 结果表明在这两种不同 条件下, $C D$ 光谱呈现了两组特征峰— $240 \mathrm{~nm}$ 处一个 负峰和 $265 \mathrm{~nm}$ 处一个正峰, 与此同时当 $\mathrm{K}^{+}$和化合物 ZSC 同时存在时特征峰也没有发生变化(图 4c). 最后熔 解温度测量实验表明 ZSC 可以使 ckit 的 TM 值升高 $6.7{ }^{\circ} \mathrm{C}$. (图 4d). 同时, 我们也对同样形成平行 $\mathrm{G}$-四联体 的 Pu22 核苷酸序列进行了研究. 结果表明, ZSC 确实可 以对其有明显的苂光响应, 荧光强度与 G-四联体的浓 度有一个很好的线性关系. 并且, 其诱导 Pu22 形成的 $\mathrm{G}$-四联体结构与 $\mathrm{K}^{+}$存在时一样具有相同的光谱性质(支
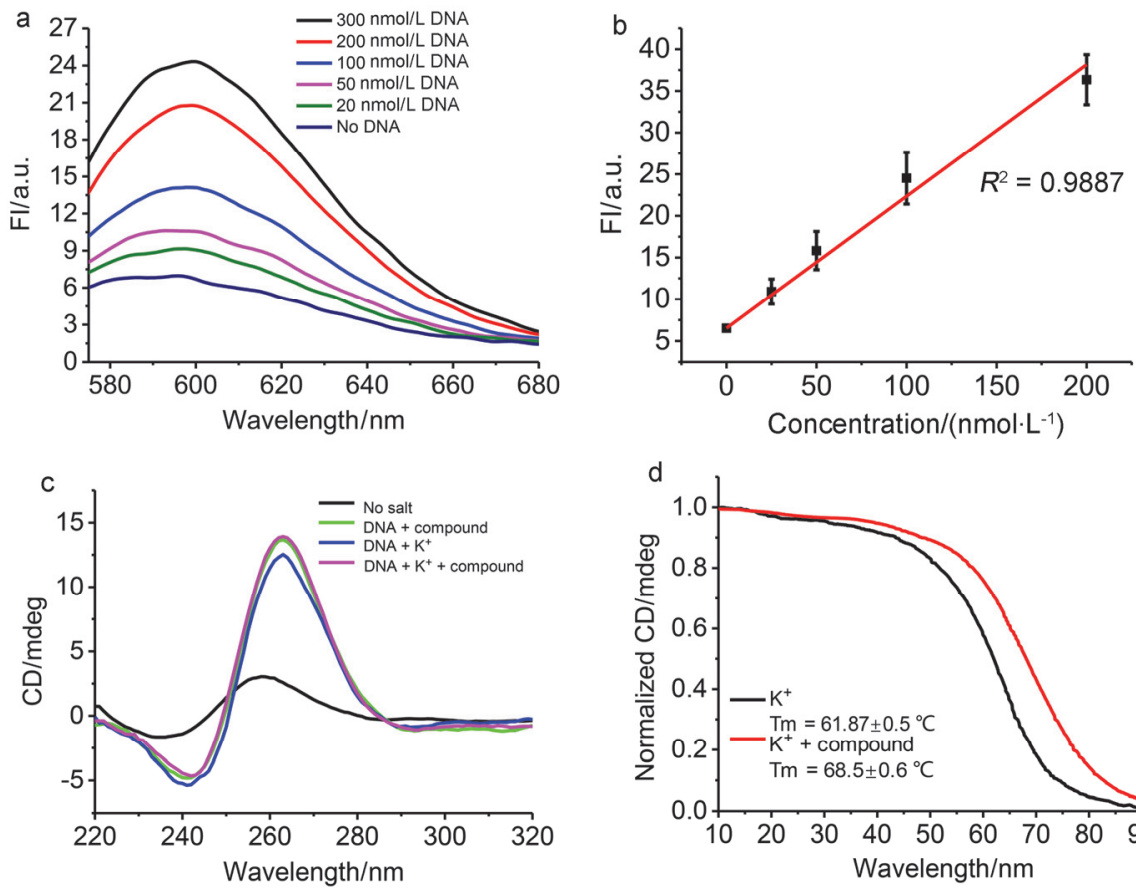

持信息图 S2).

\subsection{ZSC 与 Z 型 G-四联体 DNA 的相互作用的研究}

Phan 教授 ${ }^{[35]}$ 在 2015 年报道了左手 DNA 也称为 $\mathrm{Z}$ 型的 G-四联体的存在. 但是研究小分子化合物与 $Z$ 型 G-四联体之间的相互作用，以及调节 DNA 从 Z-型结构 到 B-型的构型转换的报道是非常少的 ${ }^{[36,37]}$. 我们选择了 $\mathrm{Z}$ 型 $\mathrm{G}-$ 四联体 $(\mathrm{ZG} 4)$ 进行了研究. 从图 $5 \mathrm{a}$ 和 $5 \mathrm{~b}$ 可以看 到, ZSC 对 ZG4 也有很好的荧光响应. 在 $\mathrm{K}^{+}$的条件下, $C D$ 光谱在 $276 \mathrm{~nm}$ 处和 $249 \mathrm{~nm}$ 处分别出现了一个负峰 和一个正峰, 这是 $Z$ 型 $\mathrm{G} 4$ 的特征峰. 值得注意的是当 体系为 $\mathrm{ZSC}$ 时, $\mathrm{CD}$ 光谱的峰发生了显著的变化出现了 平行结构 G-四联体的特征峰- - $265 \mathrm{~nm}$ 的正峰和 240 $\mathrm{nm}$ 的负峰(图 5c). 当在有 $\mathrm{K}^{+}$的体系里再加入 $\mathrm{ZSC}$ 后 $\mathrm{CD}$ 光谱的特征峰由 $\mathrm{Z}$ 型的特征峰变成了平行的特征峰 (图 5d), 这为 DNA 的构型转换提供了一定的思路.

\section{3 结论}

我们通过简单的两步反应，合成了具有诱导稳定苂 光打开检测 G-四联体的锌配体化合物. 并且通过苂光 检测发现其对 G-四联体，特别是混合型 G-四联体有一 个很强的荧光响应. 化合物与 $\mathrm{G}$-四联体之间的固有的 荧光性质使得将其用于定量检测 $\mathrm{G}$-四联体成为可能. 我们还将其作用于 $\mathrm{Z}$ 型 $\mathrm{G}$ 四联体，发现其可以将 $\mathrm{Z}$ 型 $\mathrm{G}$ 四联体变成平行结构的 $\mathrm{G}$ 四联体，并且对其也有很好的

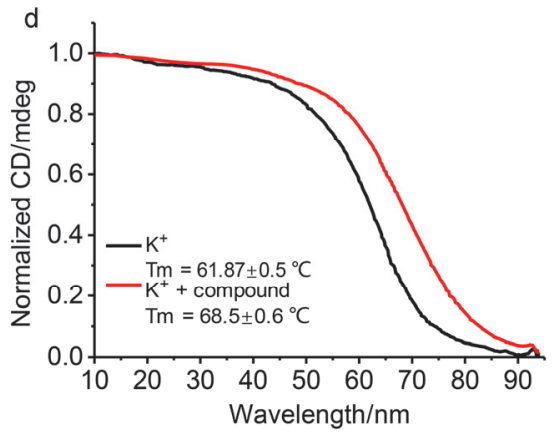

图 4 平行 $\mathrm{G}$-四联体(UspBQ1)的苂光强度图和 $\mathrm{CD}$ 分析

Figure 4 Fluorescence intensity and CD analysis of parallel G-quadruplex ckit

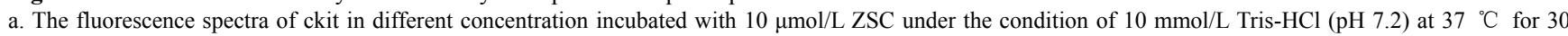
min. b. Analysis of data about fluorescence intensity dependence on different DNA concentration shown in (a). c. CD spectra of ckit under different condition. d. The melting curves of ckit under the condition of $100 \mathrm{mmol} / \mathrm{L} \mathrm{K}^{+}$and $100 \mathrm{mmol} / \mathrm{L} \mathrm{K}^{+}$with $10 \mu \mathrm{mol} / \mathrm{L} \mathrm{ZSC}$ respectively 

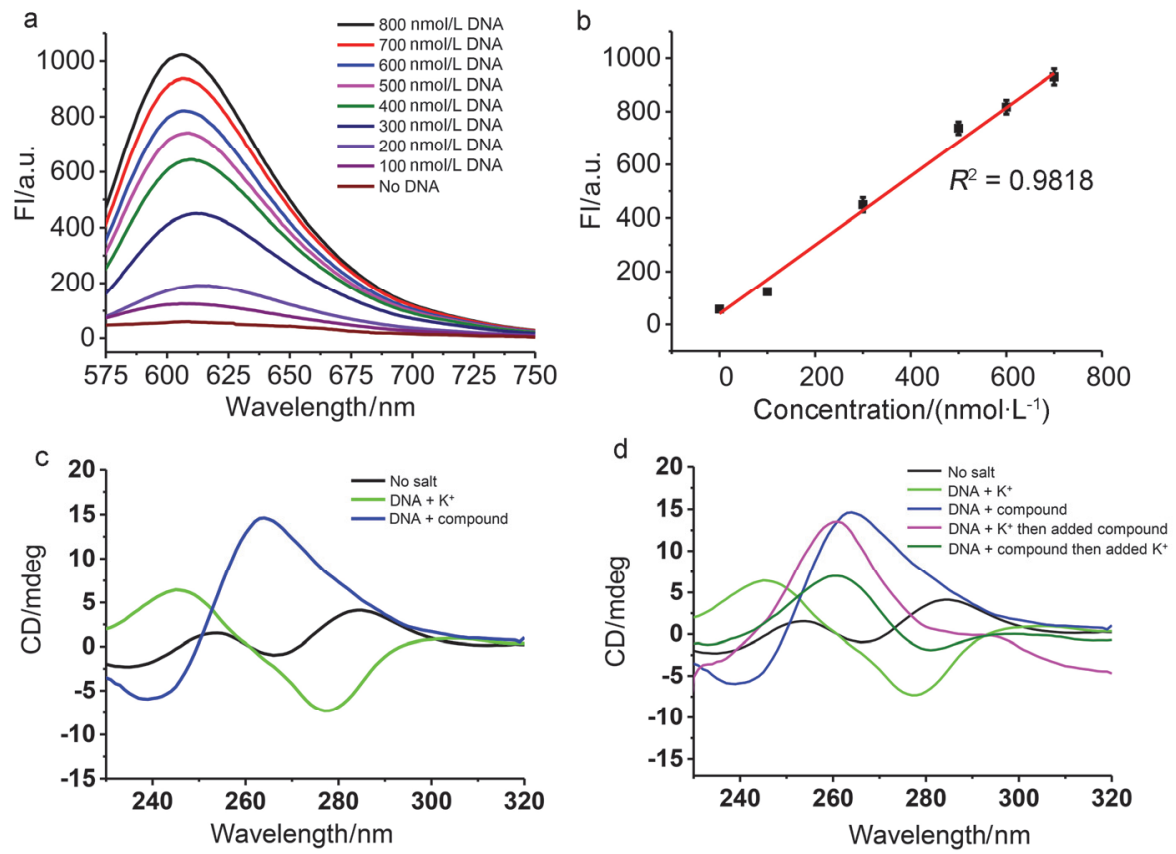

图 $5 \mathrm{Z}$ 型 $\mathrm{G}$-四联体 $(\mathrm{ZG} 4)$ 的苂光强度图和 $\mathrm{CD}$ 分析

Figure 5 Fluorescence intensity and CD analysis of Z-G-quadruplex (ZG4)

a. The fluorescence spectra of ZG4 in different concentration incubated with $10 \mu \mathrm{mol} / \mathrm{L} \mathrm{ZSC}$ under the condition of $10 \mathrm{mmol} / \mathrm{L}$ Tris- $\mathrm{HCl}(\mathrm{pH} 7.2)$ at $37{ }^{\circ} \mathrm{C}$ for 30 min. b. Analysis of data about fluorescence intensity dependence on different DNA concentration shown in (a). $\mathrm{c}$ and d. CD spectra of ZG4 under different conditions

荧光线性. 这为未来研究 DNA 不同的二级结构转变提 供了一个新思路.

\section{4 实验部分}

\section{1 主要试剂及仪器}

脱氧核糖核酸序列均购买自武汉金开瑞生物工程 有限公司(DNA 序列见支持信息 Table S1). 化学试剂均 采购自北京伊诺凯科技有限公司. 苂光仪器为 PerkinElmer LS 55 (PerkinElmer, USA). 圆二色谱信号 搜集自 Jasco-810 spectropolarimeter (Jasco, Easton, MD, USA).

\section{2 不同的 DNA 序列与 ZSC 卵受后的荧光性质的检 测}

向 $20 \mu \mathrm{L} 1 \mathrm{~mol} / \mathrm{L} \mathrm{K}^{+}$的 Tris- $\mathrm{HCl}(100 \mathrm{mmol} / \mathrm{L}, \mathrm{pH}=$ 7.2)缓冲液里加入 $100 \mu \mathrm{mol} / \mathrm{L}$ 的 DNA $(1 \mu \mathrm{L})$, 再加入 10 $\mu \mathrm{L} 200 \mu \mathrm{mol} / \mathrm{L}$ 的化合物 $\mathrm{ZSC}$, 加入 $169 \mu \mathrm{L}$ 的超纯水补 齐到 $200 \mu \mathrm{L}, 37{ }^{\circ} \mathrm{C}$ 睬育 $30 \mathrm{~min}$ 后进行荧光光谱的测试. 选择激发波长为 $540 \mathrm{~nm}$, 激发狭缝是 $15 \mathrm{~nm}$, 发射狭缝 为 $20 \mathrm{~nm}$, 扫描速度为 $500 \mathrm{~nm} / \mathrm{min}$, 从光谱图可以看到 当富 $\mathrm{G}$ 序列与化合物 $\mathrm{ZSC}$ 孵育后在 $590 \mathrm{~nm}$ 处有一个最 大的发射信号. 另外一组是在没有 $\mathrm{K}^{+}$的条件下进行的. 向 $20 \mu \mathrm{L}$ 的 Tris- $\mathrm{HCl}(100 \mathrm{mmol} / \mathrm{L}, \mathrm{pH}=7.2)$ 缓冲液里加 入 $100 \mu \mathrm{mol} / \mathrm{L}$ 的 DNA $(1 \mu \mathrm{L})$, 再加入 $10 \mu \mathrm{L} 200 \mu \mathrm{mol} / \mathrm{L}$ 的化合物 ZSC, 加入 $169 \mu \mathrm{L}$ 的超纯水补齐到 $200 \mu \mathrm{L}$, $37{ }^{\circ} \mathrm{C}$ 㽟育 $30 \mathrm{~min}$ 后进行苂光光谱的测试. 参数的设置
参考上面所述.

\section{3 不同浓度的 G-四联体与化合物 ZSC 作用后荧光 强度的研究}

向 $20 \mu \mathrm{L}$ 的 Tris- $\mathrm{HCl}(100 \mathrm{mmol} / \mathrm{L}, \mathrm{pH}=7.2)$ 缓冲液 里加入不同量的 DNA, 再加入 $10 \mu \mathrm{L} 200 \mu \mathrm{mol} / \mathrm{L}$ 的化合 物 ZSC, 加入一定体积的超纯水补齐到 $200 \mu \mathrm{L}, 37{ }^{\circ} \mathrm{C}$ 孵 育 $30 \mathrm{~min}$ 后进行苂光光谱的测试. 参数的设置参考上面 所述.

\section{4 化合物 ZSC 与 G-四联体 DNA 相互作用的 CD 光 谱}

配制 $200 \mu \mathrm{L}$ 没有 $\mathrm{K}^{+}$和化合物 ZSC 的如下反应体 系: $20 \mu \mathrm{L} 100 \mu \mathrm{mol} / \mathrm{L}$ 的 DNA, $20 \mu \mathrm{L} 100 \mu \mathrm{mmol} / \mathrm{L}$ Tris- $\mathrm{HCl}(\mathrm{pH}=7.2)$ 和 $160 \mu \mathrm{L}$ 的超纯水. 或者将 Tris- $\mathrm{HCl}$ 缓冲液换成含有 $1 \mathrm{~mol} / \mathrm{L} \mathrm{K}^{+}$的 Tris- $\mathrm{HCl}(100 \mathrm{mmol} / \mathrm{L}, \mathrm{pH}$ $=7.2$ 的条件. 含有化合物 ZSC 的反应体系如下: $20 \mu \mathrm{L}$ $100 \mu \mathrm{mol} / \mathrm{L}$ 的 DNA, $10 \mu \mathrm{L} 200 \mu \mathrm{mol} / \mathrm{L}$ 的化合物 ZSC, 20 $\mu \mathrm{L} 100 \mathrm{mmol} / \mathrm{L}$ Tris- $\mathrm{HCl}(\mathrm{pH}=7.2)$ 和 $150 \mu \mathrm{L}$ 的超纯水. 含有化合物和 $\mathrm{K}^{+}$的体系如下: $20 \mu \mathrm{L} 100 \mu \mathrm{mol} / \mathrm{L}$ 的 DNA, $10 \mu \mathrm{L} 200 \mu \mathrm{mol} / \mathrm{L}$ 的化合物 $\mathrm{ZSC}, 20 \mu \mathrm{L} 1 \mathrm{~mol} / \mathrm{L}$ $\mathrm{K}^{+}$Tris- $\mathrm{HCl}(100 \mathrm{mmol} / \mathrm{L}, \mathrm{pH}=7.2)$ 和 $150 \mu \mathrm{L}$ 的超纯水. 所有体系 $37{ }^{\circ} \mathrm{C}$ 捊育 $30 \mathrm{~min}$ 后用 Jasco-810 spectropolarimeter 获取 $\mathrm{CD}$ 光谱.

\subsection{G-四联体 DNA 熔解温度的测定}

向 $240 \mu \mathrm{L}$ 的超纯水里加入 $30 \mu \mathrm{L} 100 \mu \mathrm{mol} / \mathrm{L}$ 的 DNA, $30 \mu \mathrm{L}$ 的 $1 \mathrm{~mol} / \mathrm{L} \mathrm{K}$ 的 Tris- $\mathrm{HCl}$ 缓冲液, $37{ }^{\circ} \mathrm{C}$ 孵育 
$30 \mathrm{~min}$. 含有化合物的体系略有不同, $300 \mu \mathrm{L}$ 的体系里 含有 $225 \mu \mathrm{L}$ 的超纯水, $30 \mu \mathrm{L} 100 \mu \mathrm{mol} / \mathrm{L}$ 的 DNA, $30 \mu \mathrm{L}$ $1 \mathrm{~mol} / \mathrm{L} \mathrm{K}{ }^{+}$的 Tris- $\mathrm{HCl}(\mathrm{pH}=7.2)$ 缓冲液和 $15 \mu \mathrm{L} 200$ $\mu \mathrm{mol} / \mathrm{L}$ 的化合物 $\mathrm{ZSC}$. 同样的 $37{ }^{\circ} \mathrm{C}$ 孵育 $30 \mathrm{~min}$. 用 Jasco-810 spectropolarimeter 进行熔解温度的测定.

\subsection{2-羟基-4-(2-吡咯烷基)乙氧基苯甲醛的合成与纯 化}

在装有摚拌磁子 $250 \mathrm{~mL}$ 三口烧瓶中加入 2,4-二羟 基苯甲醛(2.76 g, $20 \mathrm{mmol}), \mathrm{N}$-(2-氯乙基)吡咯烷盐酸盐 (3.40 g, $20 \mathrm{mmol})$ 和碳酸钾 $(5.56 \mathrm{~g}, 40 \mathrm{mmol})$, 在氩气的 氛围中加入 $150 \mathrm{~mL}$ 丙酮, $60{ }^{\circ} \mathrm{C}$ 加热搅拌回流 $20 \mathrm{~h}$. 反 应结束后, 水洜减压蒸馏除去丙酮. 然后先往里加入 $200 \mathrm{~mL}$ 水, 再往里分三次, 每次加入 $200 \mathrm{~mL}$ 乙酸乙酯 萃取分液, 合并有机相, 减压蒸馏得到粗产物. 最后用 硅胶柱色谱, 淋洗剂选用 $V\left(\mathrm{CH}_{2} \mathrm{Cl}_{2}\right): V(\mathrm{CHOH})=30$ : 1 20:1 的混合液, 进行梯度淋洗. 收集产物, 真空干 燥, 得到 $3.24 \mathrm{~g}$ 灰白色固体(产率为 $68.8 \%$ ). ${ }^{1} \mathrm{H} \mathrm{NMR}$ (300 MHz, DMSO-d $) \delta$ : 9.99 (s, 1H), 7.59 (d, $J=8.6 \mathrm{~Hz}$, $1 \mathrm{H}), 6.54$ (d, $J=8.5 \mathrm{~Hz}, 1 \mathrm{H}), 6.47(\mathrm{~s}, 1 \mathrm{H}), 4.10$ (t, $J=5.1$ $\mathrm{Hz}, 2 \mathrm{H}), 2.79 \sim 2.78(\mathrm{~m}, 2 \mathrm{H}), 2.51 \sim 2.50(\mathrm{~m}, 4 \mathrm{H}), 1.66 \sim$ $1.65(\mathrm{~m}, 4 \mathrm{H}) ;{ }^{13} \mathrm{C}$ NMR (75 MHz, DMSO- $\left.d_{6}\right) \delta: 191.38$, $165.48,163.51,132.51,116.55,108.03,101.63,67.40$, 54.30, 23.45. HRMS calcd for $\mathrm{C}_{13} \mathrm{H}_{18} \mathrm{NO}_{3}{ }^{+}[\mathrm{M}+\mathrm{H}]^{+}$ 236.12812 , found 236.12787 .

\section{7 锌配体化合物(ZSC)合成与纯化}

在装有搅拌磁子的 $50 \mathrm{~mL}$ 圆底烧瓶中分别加入 2羟基-4-(2-吡咯烷基)乙氧基苯甲醛(285 mg, $1.2 \mathrm{mmol}$ )和 2,3-二氨基-2-丁烯二腈 $(65 \mathrm{mg}, 0.6 \mathrm{mmol})$. 然后加入 10 $\mathrm{mL}$ 甲醇, $60{ }^{\circ} \mathrm{C}$ 避光环境中搅拌 $20 \mathrm{~min}$; 再往反应体系 中加入二水合醋酸锌 $(132 \mathrm{mg}, 0.6 \mathrm{mmol})$, 此时溶液立即 由橘黄色变为血红色, 继续反应 $2 \mathrm{~h}$, 通过薄层色谱 $\left[V\left(\mathrm{CH}_{2} \mathrm{Cl}_{2}\right): V\left(\mathrm{CH}_{3} \mathrm{OH}\right): V\left(\mathrm{Et}_{3} \mathrm{~N}\right)=54: 3: 1\right.$ 作为展开 剂]来监测原料完全反应完毕. 反应结束后, 将有机溶 剂甲醇减压蒸馏除去, 通过硅胶柱层析分离提纯产物 [淋洗剂为 $V\left(\mathrm{CH}_{2} \mathrm{Cl}_{2}\right): V\left(\mathrm{CH}_{3} \mathrm{OH}\right): V\left(\mathrm{Et}_{3} \mathrm{~N}\right)=65: 3$ : 1]. 收集纯品, 真空干燥, 得 $245 \mathrm{mg}$ 红色固体粉末(产率 为 $67.7 \%) .{ }^{1} \mathrm{H} \mathrm{NMR}\left(300 \mathrm{MHz}, \mathrm{DMSO}-d_{6}\right) \delta: 8.35(\mathrm{~s}, 2 \mathrm{H})$, $7.46 \sim 7.15(\mathrm{~m}, 2 \mathrm{H}), 6.23 \sim 6.22(\mathrm{~m}, 4 \mathrm{H}), 4.12 \sim 4.11(\mathrm{~m}$, $4 \mathrm{H}), 2.98 \sim 2.72(\mathrm{~m}, 4 \mathrm{H}), 2.71 \sim 2.34(\mathrm{~m}, 8 \mathrm{H}), 1.99 \sim 1.54$ $(\mathrm{m}, 8 \mathrm{H}) ;{ }^{13} \mathrm{C}$ NMR $\left(75 \mathrm{MHz}, \mathrm{DMSO}-d_{6}\right) \delta: 176.89,166.50$, $161.21,138.27,120.96,114.69$, 112.40, 107.56, 105.05, 67.07, 54.35, 23.50. HRMS calcd for $\mathrm{C}_{30} \mathrm{H}_{33} \mathrm{~N}_{6} \mathrm{O}_{4} \mathrm{Zn}^{+}[\mathrm{M}$ $+\mathrm{H}]^{+}$605.18493, found 605.18161 .

\section{References}

[1] Bhasikuttan, A. C.; Mohanty, J. Chem. Commun. 2015, 51, 7581.

[2] Liu, C.; Chen, Y.; Wang, Y.; Wu, F.; Zhang, X.; Yang, W.; Wang, J.; Chen, Y.; He, Z.; Zou, G.; Wang, S.; Zhou, X. Nano Res. 2017, DOI: 10.1007/s12274-017-1445-2.
[3] Liu, C.; Wang, Y.; Zhang, X.; Wu, F.; Yang, W.; Zou, G.; Yao, Q.; Wang, J.; Chen, Y.; Wang, S.; Zhou, X. Chem. Sci. 2017, DOI: 10.1039/C7SC00637C.

[4] Tian, T.; Xiao, H.; Weng, X.; Wang, S.; Zhou, X. Sci. Sin. Chim. 2013, 42, 1700 (in Chinese). (田沺, 肖街, 翁小成, 王少儒, 周翔, 中国科学: 化学, 2013, 42, 1700.)

[5] Biffi, G.; Tannahill, D.; McCafferty, J.; Balasubramanian, S. Nat. Chem. 2013, 5, 182 .

[6] Chen, S. B.; Hu, M. H.; Liu, G. C.; Wang, J.; Ou, T. M.; Gu, L. Q.; Huang, Z. S.; Tan, J. H. J. Am. Chem. Soc. 2016, 138, 10382.

[7] Wang, S.; Min, Y.; Wang, J.; Liu, C.; Fu, B.; Wu, F., Wu, L; Qiao, Z.; Song, Y; Xu, G.; Wu, Z.; Huang, G.; Peng, N.; Huang, R.; Mao, W.; Peng, S.; Chen, Y.; Zhu, Y.; Tian, T.; Zhang, X.; Zhou, X. Sci. Adv. 2016, 2, e1501535

[8] Wang, S.; Zhang, Q.; Wang, J.; Ge, X.; Song, Y.; Wang, Y.; Li, X.; Fu, B.; Xu, G.; Shu, B.; Gong, P.; Zhang, B.; Tian, T.; Zhou, X. Cell Chem. Biol. 2016, 23, 1113.

[9] Zheng, X. H; Mu, G.; Tan, C. P.; Cao, G.; Mao, Z. W. Sci. Sin. Chim. 2014, 44, 484 (in Chinese). (郑小辉, 穆舸, 谭彩萍, 曹乾, 毛宗 万，中国科学: 化学 $2014,44,484$.)

[10] Lin, S.; Xu, M.; Yuan, G. Chin. Chem. Lett. 2012, 23, 329.

[11] Yan, J.; Guan, Y. Progr. Anatom. Sci. 2015, 21, 419.

[12] Zheng, X. Liu, H.; Xia, L; Mao, Z. Chin. Pharmacol. Bull. 2016, 32, 751 (in Chinese). (郑小辉, 刘海英, 夏立新, 毛宗万, 中国药 理学通报, 2016, 32, 751.)

[13] Müller, S.; Kumari, S.; Rodriguez, R.; Balasubramanian, S. Nat. Chem. 2010, 2, 1095.

[14] Zhang, C.; Wu, W.; Lu, Y.; Gu, L.; Huang, Z. S. Acta Chim. Sinica 2008, 66, 953 (in Chinese). (张弛, 吴伟涁, 卢宇靖, 古练权, 黄志 纾, 化学学报, 2008, 66, 953.)

[15] Zheng, K.; Zhang, D.; Zhang, L.; Hao, Y.; Zhou, X.; Tan, Z. J. Am. Chem. Soc. 2011, 133, 1475.

[16] Ou, Z. Z.; Ju, B. L.; Gao, Y. Y.; Wang, Z. C.; Huang, G.; Qian, Y. M. Acta Phys.-Chim. Sin. 2015, 31, 2386 (in Chinese). (欧植泽，句宝 龙, 高云燕, 王子超, 黄干, 钱一梦, 物理化学学报, 2015, 31, 2386.)

[17] Meng, C.; Wang, S.; Zhang, K.; Jin, H.; Chen, X.; Hu, M.; Xiong, J. Acta Chim. Sinica 2011, 69, 1173 (in Chinese). (孟晨鹏, 王舜, 张 克军, 金辉乐, 陈锡安, 胡茂林, 熊静, 化学学报, 2011, 69, 1173.)

[18] Reed, J. E.; Arnal, A. A.; Neidle, S.; Vilar, R. J. Am. Chem. Soc. 2006, 128, 5992.

[19] Xu, L.; Wu, W.; Ding, J.; Feng, S.; Xing, X.; Deng, M.; Zhou, X. RSC Adv. 2012, 2, 894.

[20] Yu, L.; Gai, W.; Yang, Q.; Xiang, J.; Sun, H.; Li, Q.; Wang, L.; Guan, A.; Tang, Y. Chin. Chem. Lett. 2015, 26, 705.

[21] Wu, F.; Liu, C.; Chen, Y.; Yang, S.; Xu, J.; Huang, R.; Wang, X.; Li, M.; Liu, W.; Mao, W.; Zhou, X. Sens. Actuators, B 2016, 236, 268.

[22] Tera, M.; Iida, K.; Ikebukuro, K.; Seimiya, H.; Shin-ya, K.; Nagasawa, K. Org. Biomol. Chem. 2010, 8, 2749.

[23] Chen, Y.; Yan, S.; Yuan, L.; Zhou, Y.; Song, Y.; Xiao, H.; Weng, X.; Zhou, X. Org. Chem. Front. 2014, 1, 267.

[24] Xu, S.; Li, Q.; Xiang, J.; Yang, Q.; Sun, H.; Guan, A.; Wang, L.; Liu, Y.; Yu, L.; Shi, Y.; Chen, H.; Tang, Y. Nucleic Acids Res. 2015, 43, 9575.

[25] Mohanty, J.; Barooah, N.; Dhamodharan, V.; Harikrishna, S.; Pradeepkumar, P. I.; Bhasikuttan, A. C. J. Am. Chem. Soc. 2013, 135, 367.

[26] Chen, S. B.; Wu, W. B.; Hu, M. H.; Ou, T. M.; Gu, L. Q.; Tan, J. H.; Huang, Z. S. Chem. Commun. 2014, 50, 12173.

[27] Lin, S.; Gao, W.; Tian, Z.; Yang, C.; Lu, L.; Mergny, J.; Leung, C.; Ma, D. Chem. Sci. 2015, 6, 4284.

[28] Ji, X.; Yao, C.; Wan, Y.; Song, H.; Xin, P.; Cui, H.; Zheng, C.; Deng, S. Chin. J. Chem. 2016, 34, 331 .

[29] Liu, X.; Yang, Y.; Hua, X.; Feng, X.; Su, S.; Huang, Y.; Fan, Q.; Wang, L.; Huang, W. Chin. J. Chem. 2015, 33, 981.

[30] Shao, Y.; Li, C.; Zhou, X.; Chen, P.; Yang, Z.; Li, Z.; Liu, D. Acta Chim. Sinica 2015, 73, 815 (in Chinese). (邵昱, 李闯, 周旭, 陈平, 杨忠强, 李志波, 刘冬生, 化学学报, 2015, 73, 815.)

[31] Liu, X.; Wang, Y.; Huang, Y.; Feng, X.; Fan, Q.; Huang, W. Acta Chim. Sinica 2016, 74, 664 (in Chinese). (刘兴奋, 王亚腾, 黄艳 琴, 冯晓苗, 范曲立, 黄维, 化学学报, 2016, 74, 664.)

[32] Zhou, J.; Yuan, G. Acta Chim. Sinica 2007, 65, 1728 (in Chinese). (周江, 袁谷, 化学学报, 2007, 65, 1728.)

[33] Wei, Q.; Qu, F.; Gong, R. Prog. Biochem. Biophys. 2015, 42, 624 (in Chinese). (魏强, 屈锋, 龚苪, 生物化学与生物物理进展, 2015, 


$$
42,624 \text {.) }
$$

[34] Rankin, S.; Reszka, A. P.; Huppert, J.; Zloh, M.; Parkinson, G. N.; Todd, A. K.; Ladame, S.; Balasubramanian, S.; Neidle, S. J. Am. Chem. Soc. 2005, 127, 10584.

[35] Chung, W. J.; Heddi, B.; Schmitt, E.; Lim, K. W.; Mechulam, Y.;
Phan, A. T. Proc. Natl. Acad. Sci. U. S. A. 2015, 112, 2729

[36] Zhao, A.; Zhao, C.; Ren, J.; Qu, X. Chem. Commun. 2016, 52, 1365

[37] Fu, B.; Huang, J.; Chen, Y.; Wang, Y.; Xue, T.; Xu, G.; Wang, S.; Zhou, X. Chem. Commun. 2016, 52, 10052

(Cheng, F.) 